\title{
Assembly, Function and Evolution of Cyanobacterial Carboxysomes
}

\author{
Cheryl A. Kerfeld ${ }^{1,2,3,4}$ and Matthew R. Melnicki ${ }^{3,4}$
}

${ }^{1}$ MSU-DOE Plant Research Laboratory, Michigan State University, East Lansing, MI 48824, USA

${ }^{2}$ Department of Biochemistry and Molecular Biology, Michigan State University, East Lansing, MI 48824, USA

${ }^{3}$ Physical Biosciences Division, Lawrence Berkeley National Laboratory, Berkeley, CA 94720, USA

${ }^{4}$ Department of Plant and Microbial Biology, University of California, Berkeley, Berkeley, CA 94720, USA

\section{SHORT TITLE:}

Assembly, Function and Evolution of Carboxysomes

\begin{abstract}
All cyanobacteria contain carboxysomes, RuBisCO-encapsulating bacterial microcompartments that function as prokaryotic organelles. The two carboxysome types, alpha and beta, differ fundamentally in components, assembly, and species distribution. Alpha carboxysomes share a highly-conserved gene organization, with evidence of horizontal gene transfer from chemoautotrophic proteobacteria to the picocyanobacteria, and seem to co-assemble shells concomitantly with aggregation of cargo enzymes. In contrast, beta carboxysomes assemble an enzymatic core first, with an encapsulation peptide playing a critical role in formation of the surrounding shell. Based on similarities in assembly, and phylogenetic analysis of the pentameric shell protein conserved across all bacterial microcompartments, beta carboxysomes appear to be more closely related to the microcompartments of heterotrophic bacteria (metabolosomes) than to alpha carboxysomes, which appear deeply divergent. Beta carboxysomes can be found in the basal cyanobacterial clades that diverged before the ancestor of the chloroplast and have recently been shown to be able to encapsulate functional RuBisCO enzymes resurrected from ancestrally-reconstructed sequences, consistent with an ancient origin. Alpha and beta carboxysomes are not only distinct units of evolution, but are now emerging as genetic/metabolic modules for synthetic biology; heterologous expression and redesign of both the shell and the enzymatic core have recently been achieved.
\end{abstract}

(C) 2016. This manuscript version is made available under the Elsevier user license

http://www.elsevier.com/open-access/userlicense/1.0/ 


\section{INTRODUCTION}

\section{Carboxysomes: metabolic modules for $\mathrm{CO}_{2}$ fixation}

More than 50 years ago, unusual polyhedral bodies were discovered in cyanobacteria by electron microscopy [1] (Figure 1A-C). They were subsequently identified as proteinaceous shells that were packed with the $\mathrm{CO}_{2}$-fixation enzyme ribulose 1,5-bisphosphate carboxylase/oxygenase (RuBisCO) and, accordingly, named carboxysomes [2]. Through identification of homologs of the delimiting shell proteins in microbial genomes, it is now known that carboxysomes are members of a class of architecturallyrelated structures, bacterial microcompartments (BMCs). The potential to form BMCs has been found in at least 23 bacterial phyla, where they play diverse roles in metabolism dictated by the function of the encapsulated enzymes $[3,4]$. The widespread distribution of BMCs in bacteria suggests frequent horizontal gene transfer of these genetic and metabolic modules [3-5]. However, despite their functional diversity, only two types of BMC are known to be involved in autotrophic metabolism (specifically, the Calvin-Benson Cycle): the alpha and beta carboxysomes, which are found in many chemoautotrophic bacteria, some purple-sulfur phototrophs, and all cyanobacteria. Among the myriad of BMC types, only carboxysomes have been identified in the Cyanobacteria.

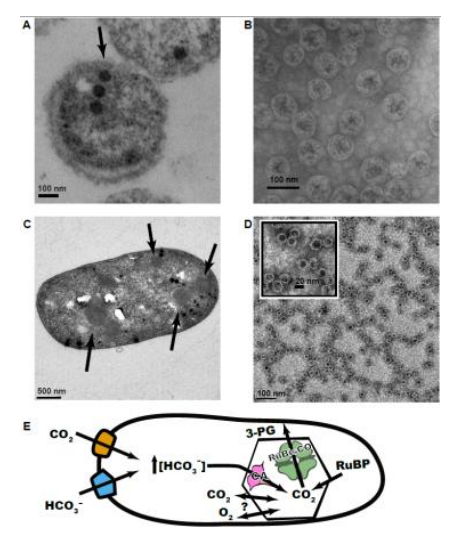

Figure 1. Carboxysomes and the cyanobacterial CCM.

A. TEM of Prochlorococcus MED4 (Image courtesy of Drs. Sarah Bagby, Nicki Watson, and Penny Chisholm). B. Purified Prochlorococcus MED4 alpha carboxysomes (courtesy of Drs. Sabine Heinhorst and Gordon Cannon). C. TEM of Halothece sp. PCC 7418. D. Empty beta carboxysome shells (from Halothece PCC 7418) expressed in and purified from E. coli [6]. E. Schematic of the cyanobacterial CCM. RuBP = ribulose 1,5-bisphosphate; 3-PG = 3phosphoglycerate; $\mathrm{CA}=$ carbonic anhydrase.

The carboxysome is the core component of the cyanobacterial Carbon Concentrating Mechanism (CCM) (Figure 1E) [7-9] that also involves the active uptake of inorganic carbon into the cell and its intracellular accumulation primarily as $\mathrm{HCO}_{3}{ }^{-}[10,11]$. $\mathrm{HCO}_{3}{ }^{-}$diffuses through the carboxysome shell; within the lumen, an encapsulated carbonic anhydrase (CA) converts it into $\mathrm{CO}_{2}$, promoting the carboxylation reaction of RuBisCO. Studies of carboxysomal proteins provided the first structural examples of the ubiquitous BMC shell proteins, the hexamers (BMC-H), pseudohexamers (BMC-T), and pentamers (BMCP) that assemble into an apparently icosahedral shell [12-14] (Figure 2). Hexamers constitute the predominant building blocks of the shell; these proteins are typically perforated by a small pore at the symmetry axis that presumably allows polar molecules such as bicarbonate to pass [15]. Experimentally, it has been shown that the shell provides diffusional resistance to $\mathrm{CO}_{2}$ [16], preventing its loss from inside 
the carboxysome [17], and possibly provides a barrier to $\mathrm{O}_{2}$, minimizing the unproductive oxygenation reaction of RuBisCO.
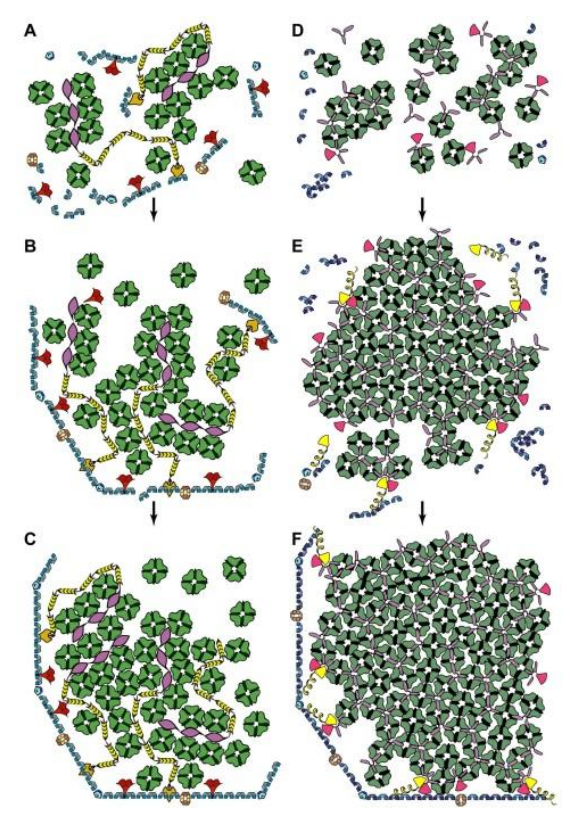

Figure 2. Carboxysome assembly.

A,B,C. Alpha carboxysome shells begin assembling concomitantly with aggregation of RuBisCO. D,E,F. Beta carboxysome shells begin assembling after RuBisCO aggregation has formed a densely-packed paracrystalline array. Symbols for individual components are defined in Table 1.

\section{Alpha and beta carboxysomes are distinct BMCs}

Although alpha and beta carboxysomes share similar general architectural and functional principles - a selectively permeable protein shell that encapsulates RuBisCO with CA - they differ in many details, including the type of RuBisCO (Form 1A in alpha, Form 1B in beta). The "core" operons (Table 1) for alpha (cso) and beta ( $\mathrm{ccm}$ ) carboxysomes differ in the form of carbonic anhydrase encapsulated (CsoSCA, a beta-class CA; CcmM, a gamma-class CA). In addition to CcmM, a subset of cyanobacterial species also package an additional beta-class CA encoded at a "satellite" locus outside of the $\mathrm{ccm}$ operon [18]. This protein, CcaA, is evolutionarily distant from CsoSCA; the only residues conserved between their primary structures are the $\mathrm{Zn}$-coordinating residues of the active sites [13]. Finally, the alpha and beta core operons each contain an additional gene for a protein of unknown function: the $\sim 850$ amino acid protein CsoS2 and the $\sim 230$ amino acid protein $\mathrm{CcmN}$, respectively. These two proteins have no sequence homology and their functions, other than being critical for carboxysome assembly, remain enigmatic [19-23].

In freshwater and terrestrial beta cyanobacterial species in which beta carboxysomes are prevalent, the ccm genes are encoded in the ccmKLMN operon, but genes for other components, some essential, are scattered throughout the genome in "satellite" loci (Table 1; recently reviewed in [3]). For example, in many organisms the genes for RuBisCO are encoded remotely from the $\mathrm{ccm}$ operon. Beta carboxysomes have not yet been purified to homogeneity, and other structural components may yet be identified; for example, comparative genomic evidence suggests that RuBisCO activase may be localized 
to beta carboxysomes [18]. In contrast, the alpha cyanobacteria, which includes marine Synechococcus and Prochloroccus clades, contain the canonical cso operon, which has recently been shown to be nested in a "superlocus" which contains other structural components of the alpha carboxysome $[3,24]$. For example, $\operatorname{csoS1D}$, which encodes a member of a distinct clade of BMC-T proteins that form stacked trimers with gated apertures $[12,25]$, is encoded upstream of the cso operon in all alpha cyanobacteria. Computational analysis using all available Cyanobacteria genomes indicates that both the $\mathrm{ccm}$ and cyanobacterial cso superloci also contain widely conserved genes for ancillary or co-regulated functions, such as components of membrane-bound inorganic carbon uptake systems (Table 1) [3,24].

\section{Assembly of alpha and beta carboxysomes}

The distinctive structural components of the alpha and beta carboxysomes are likely related to fundamental differences in their modes of assembly (Figure 2). Cryo-electron tomography studies on Halothiobacillus neapolitanus, the chemoautotrophic model organism for alpha carboxysome research [26], revealed partially assembled shells with attached RuBisCO molecules [27], suggesting a coassembly pathway for shell and cargo proteins. Indeed some of the internal, core proteins appear to be integrated into the alpha carboxysome shell, including CsoSCA [28-30]. Alpha carboxysomes are the only type of BMC for which it has been established that an intrinsically disordered protein (IDP) - CsoS2 - plays a key role in assembly and function [20]. IDPs are known to alter their conformation, including acquiring secondary and tertiary structure, in the presence of their binding partners [31,32]. Small Angle $\mathrm{X}$-ray Scattering analysis has shown that, in the presence of RuBisCO, CsoS2 adopts a more compact fold and interacts with both RuBisCO and the shell [20]. CsoS2 is the third most abundant protein in the alpha carboxysome and is essential to carboxysome formation, as deletion mutants lack carboxysomes [20]. In contrast, in RuBisCO deletion mutants of alpha cyanobacteria, shell structures are still observed, resembling wildtype alpha carboxysomes in shape and size [26].

In beta cyanobacteria there is no evidence for formation of empty carboxysomes/shells when core components of the carboxysome are deleted. However when the shell proteins are expressed in E. coli, empty polyhedral shells are formed [6], however they are considerably smaller ( $20 \mathrm{~nm})$ than their packed counterparts (Figures 1C \& 1D). Beta carboxysomes have been shown to assemble from the inside out; initially, soluble RuBisCO is nucleated by domains of the $\mathrm{CcmM}$ protein that resemble the small subunit of RuBisCO [33-36] (Figure 2D). The N-terminal gamma-class carbonic anhydrase domain of CcmM interacts with both the shell [6] and with the N-terminal domain of $\mathrm{CcmN}$. In turn, $\mathrm{CcmN}$ contains a C-terminal encapsulation peptide, which is typically 15-20 amino acids [19]. This region, which is predicted to form an amphipathic alpha helix that interacts with the shell, is essential for the assembly of the shell around the catalytic core (Figure 2E) [19]. CcmN mutants lacking this extension do not form carboxysomes [19]. Notably, encapsulation peptides have been identified in all of the experimentally 
characterized types of BMCs, including heterotrophic "metabolosomes", with the notable exception of the alpha carboxysome $[19,37,38]$.

\section{Evolution of carboxysomes}

For large multiprotein complexes, assembly pathways frequently reflect their evolution $[39,40]$. Given the similarities among the structural features that are essential for assembly [4], we suggest that beta carboxysomes are more closely related to the metabolosomes (heterotrophic BMCs) than to the alpha carboxysomes. This inference is supported by a phylogenetic analysis of all BMC-P shell protein sequences (Figure 3). The alpha cyanobacterial sequences (for both the conserved CsoS4A and CsoS4B paralogs) are nested among the BMC-P sequences from the alpha carboxysome loci of chemoautotrophs. Moreover, it is clear that the alpha CsoS4 proteins are evolutionarily distant from all other BMC-P sequences. In contrast, the $\mathrm{CcmL}$ proteins of beta carboxysomes form a group clustered among the BMC-P proteins from diverse metabolosomes. This provides evidence of a deep divergence between the two types of carboxysomes.

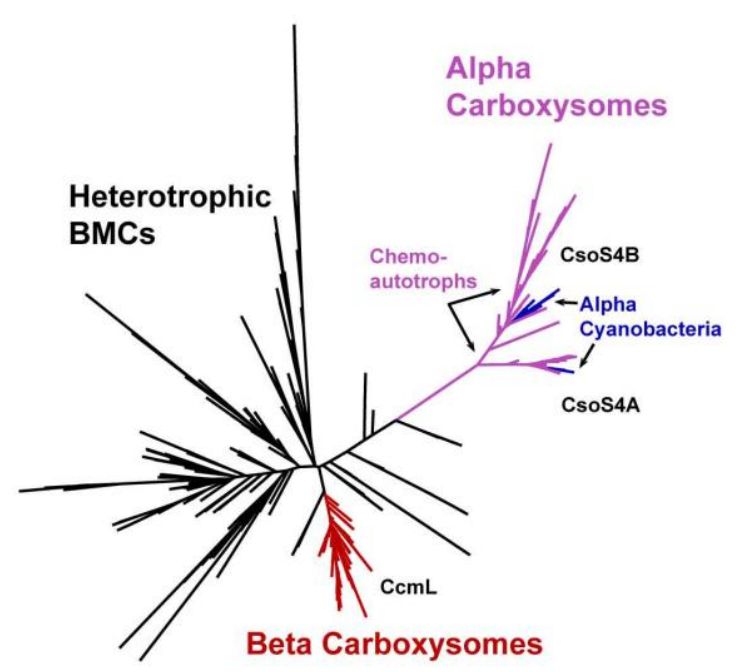

Figure 3. Evolution of alpha and beta carboxysomal BMC-Ps.

All BMC-P amino acid sequences were taken from the RP75 database scored with the PF03319 Pfam HMM for BMC-P domains, aligned in MUSCLE, made nonredundant to $95 \%$, and used to build trees in PhyML.

These observations raise the question of how the two types evolved. Among all BMCs, sequence homology can be detected for both classes of shell proteins (BMC-P \& BMC-H/T), implicating a common ancestry for the shell. Previously, it had been speculated that beta carboxysomes arose by shell recruitment (to encapsulate already-aggregating RuBisCO-CA complexes) whereas alpha carboxysomes arose by "invasion" of a heterotrophic BMC by RuBisCO [41]. It is clear that the alpha carboxysomes of Cyanobacteria share ancestry with their chemoautotrophic counterparts (which are predominantly found in Proteobacteria), as both groups maintain the core gene order of the cso locus (cbbL, cbbS, csoS2, $\operatorname{csoS3}, \operatorname{csoS} 4 A, \operatorname{csoS4B})[3,20]$. The extent of this conservation between two evolutionarily distant phyla implies horizontal gene transfer. Notably, genetic markers showing strong evidence for horizontal gene 
transfer between proteobacteria and alpha cyanobacteria have recently been found, although the directionality of this transfer was unclear [42]. From the BMC-P phylogeny (Figure 3) as well as that of CsoS2 [20], it appears that the alpha cyanobacteria most likely were the recipients of a transfer event which included the CsoS4A/B and CsoS2 genes. Given the conservation of proximal components, this would suggest that the whole cyanobacterial alpha carboxysome operon was derived in this manner.

As with studies of the early events of cyanobacterial evolution, it is difficult to predict how beta carboxysomes arose in cyanobacteria. The incorporation of RuBisCO into carboxysomes appears to be sensitive to its specific form; for example, Form II RuBisCO cannot be encapsulated in either alpha $[26,43]$ or beta carboxysomes $[44,45]$. Even more strikingly, only one of the two Form $1 \mathrm{~A}$ RuBisCOs, the one associated with the carboxysome locus, encoded in the chemoautotroph Thiomicrospira crunogena, can be encapsulated in alpha carboxysomes [26]. However, computationally-reconstructed ancestral RuBisCO genes have recently been expressed and shown to assemble into functional $\mathrm{L}_{8} \mathrm{~S}_{8}$ complexes with similar kinetics [46]. Moreover, the large subunits for the resurrected forms (ancestral Form 1A, ancestral Form 1B) could be encapsulated by extant beta carboxysomes, suggesting an ancient origin for both types of carboxysomes.

While even the basal cyanobacterial genus, Gloeobacter, contains beta carboxysomes, genes for BMC shell proteins cannot be detected in genomes of the Melainabateria, a new class of aphotic bacteria which are basal but monophyletic with the cyanobacteria [47] (although these were sequenced with culture-independent methods and, accordingly, may have assembly errors or incomplete coverage). In contrast, genes for shell proteins (presumably components of metabolosomes) have been found in all phyla that include photosynthetic prokaryotes (Chlorobi, Proteobacteria, Chloroflexi, Firmicutes, Acidobacteria, and the recently described Gemmatimonadetes) [3]. It is clear that the photosynthetic machinery (particularly for the two photosystems) must have been transferred horizontally at least once from these organisms to the cyanobacteria [48]. As metabolosome-associated BMC genes tend to be frequently transferred horizontally, often as entire operons [3-5], it is plausible that carboxysome shell proteins were acquired in an analogous (or even the same) transfer event.

There are several arguments which suggest that carboxysomes might have originated as recently as the Phanerozoic ( 0.4 giga-annum $(\mathrm{Ga})$ ), when global $\mathrm{CO}_{2}$ concentrations are predicted to have dropped and $\mathrm{O}_{2}$ concentrations had begun to approach present levels [46]. This would place the development of carboxysomes after the primary endosymbiosis event which led to the development of the chloroplast [49], and could explain the absence of carboxysomes from plants and algae. Likewise, the presence of homologous photorespiratory salvage pathways in cyanobacteria and chloroplasts has been taken to suggest a relatively late development of the CCM. However, it is puzzling that some subclades of extant cyanobacteria, such as Gloeobacter (subclade G as defined in [50]) and Pleurocapsa (subclade F as 
defined in [50]), contain beta carboxysomes despite having branched off before the ancestor of the plant/algal chloroplast $[49,50]$. Were these genes acquired afterwards? Or perhaps carboxysomes were already present in some cyanobacterial lineages, but not in the endosymbiont. Because CCMs are able to promote calcium mineralization as a consequence of bicarbonate uptake, it has been speculated that carboxysomes could have facilitated cyanobacterial stromatolite formation, fossils dating from as far back as the early Proterozoic (2.5 Ga) [51]. Fossilized akinetes from specimens resembling those derived from filamentous heterocystous bacteria have recently been observed in cherts as old as $2.1 \mathrm{Ga}$ [52], thereby providing a calibration point for a late step in the evolution of cyanobacteria. If carboxysomes had arisen this far back in geologic history, perhaps there was another functional advantage besides facilitation of $\mathrm{CO}_{2}$ fixation and avoidance of photorespiration.

\section{Prospects}

BMCs can be considered units of evolution, with their broad species distribution, conserved locus structure, and considerable evidence for their frequent horizontal gene transfer [3-5]. These features likewise are establishing BMC loci as units of engineering, when viewed as genetically-encoded metabolic modules which can be transferred for plug-and-play applications in synthetic biology [53]. For example, the canonical cso operon for the alpha carboxysome has been expressed in E. coli, yielding structures with demonstrable RuBisCO activity [54]. Likewise, given the speculation that RuBisCO has already been maximally optimized by evolution $[55,56]$, efforts are underway to install carboxysomes into chloroplasts in order to enhance crop productivity and nitrogen utilization efficiency $[57,58]$. Parallel efforts to re-engineer the carboxysome based on knowledge of protein-protein interactions involved in carboxysome assembly, first simplifying it genetically for facile transfer into diverse organisms, provides proof-of-concept of a domain fusion approach for engineering designed BMCs with novel functions [59]. These achievements not only substantiate our current understanding of carboxysome structure, function, and assembly, they also portend a new era in which industrially-relevant pathways could be encapsulated in proteinaceous organelles, recapitulating the ancient evolutionary innovation of compartmentalization of photosynthesis by endosymbiosis.

\section{ACKNOWLEDGEMENTS}

We thank members of the Kerfeld lab for engaging discussions, Dr. C. Raul Gonzalez for electron micrographs of cyanobacteria, and Dr. Fei Cai for micrographs of purified carboxysome shells and for a critical reading and assistance in revising this manuscript. This work was supported by the Office of Science of the U.S. Department of Energy DE-FG02-91ER20021 and with infrastructure support from MSU AgBio Research. 


\section{REFERENCES AND RECOMMENDED READING}

* of special interest

${ }^{\star *}$ of outstanding interest

1. Drews G, Niklowitz W: Cytology of Cyanophycea. II. Centroplasm and granular inclusions of Phormidium uncinatum Archiv fur Mikrobiologie 1956, 24.

2. Shively JM, Ball F, Brown DH, Saunders RE: Functional organelles in prokaryotes: polyhedral inclusions (carboxysomes) of Thiobacillus neapolitanus. Science 1973, 182:584-586.

** 3. Axen SD, Erbilgin O, Kerfeld CA: A taxonomy of bacterial microcompartment loci constructed by a novel scoring method. PLoS Comput Biol 2014, 10:e1003898. ** This paper provides a comprehensive study of all BMC types, and helped to establish which accessory genes are conserved across carboxysome loci as well as identified satellite loci, and strengthened the concept of a cso "superlocus".

4. Kerfeld CA, Erbilgin O: Bacterial microcompartments and the modular construction of microbial metabolism. Trends Microbiol 2015, 23:22-34.

5. Lawrence JG, Roth JR: Selfish Operons: Horizontal Transfer May Drive the Evolution of Gene Clusters. Genetics 1996, 143:1843-1860.

6. Cai F, Bernstein SL, Wilson SC, Kerfeld CA: Production and Characterization of Synthetic Carboxysome Shells with Incorporated Luminal Proteins. Plant Physiology 2016, 170.

7. Badger MR, Price GD: The $\mathbf{C O}_{2}$ concentrating mechanism in cyanobacteria and microalgae. Physiol Plant 1992, 90:529-536.

8. Kaplan A, Reinhold L: $\mathbf{C O}_{2}$ concentrating mechanisms in photosynthetic microorganisms. Annu Rev Plant Physiol Plant Mol Biol 1999, 50:539-570.

9. Price GD, Badger MR, Woodger FJ, Long BM: Advances in understanding the cyanobacterial $\mathrm{CO}_{2}$-concentrating-mechanism (CCM): functional components, $\mathrm{Ci}$ transporters, diversity, genetic regulation and prospects for engineering into plants. J Exp Bot 2008, 59:1441-1461.

10. Dobrinski KP, Longo DL, Scott KM: The carbon-concentrating mechanism of the hydrothermal vent chemolithoautotroph Thiomicrospira crunogena. Journal of Bacteriology 2005, 187:5761-5766.

11. Price GD, Sultemeyer D, Klughammer B, Ludwig M, Badger MR: The functioning of the $\mathrm{CO}_{2}$ concentrating mechanism in several cyanobacterial strains: a review of general physiological characteristics, genes, proteins and recent advances. Can $J$ Bot 1998, 76:973-1002.

12. Klein MG, Zwart P, Bagby SC, Cai F, Chisholm SW, Heinhorst S, Cannon GC, Kerfeld CA: Identification and Structural Analysis of a Novel Carboxysome Shell Protein with Implications for Metabolite Transport. Journal of Molecular Biology 2009, 392:319333.

13. Sawaya MR, Cannon GC, Heinhorst S, Tanaka S, Williams EB, Yeates TO, Kerfeld CA: The structure of beta-carbonic anhydrase from the carboxysomal shell reveals a distinct subclass with one active site for the price of two. Journal of Biological Chemistry 2006, 281:7546-7555.

14. Tanaka S, Kerfeld CA, Sawaya MR, Cai F, Heinhorst S, Cannon GC, Yeates TO: Atomiclevel models of the bacterial carboxysome shell. Science 2008, 319:1083-1086. 
15. Kerfeld CA, Sawaya MR, Tanaka S, Nguyen CV, Phillips M, Beeby M, Yeates TO: Protein structures forming the shell of primitive bacterial organelles. Science 2005, 309:936-938.

16. Dou Z, Heinhorst S, Williams EB, Murin CD, Shively JM, Cannon GC: $\mathbf{C O}_{2}$ fixation kinetics of Halothiobacillus neapolitanus mutant carboxysomes lacking carbonic anhydrase suggest the shell acts as a diffusional barrier for $\mathbf{C O}_{2}$. J Biol Chem 2008 , 283:10377-10384.

17. Cai F, Menon BB, Cannon GC, Curry KJ, Shively JM, Heinhorst S: The Pentameric Vertex Proteins Are Necessary for the Icosahedral Carboxysome Shell to Function as a $\mathrm{CO}_{2}$ Leakage Barrier. PLOS ONE 2009, 4:e7521.

18. Zarzycki J, Axen SD, Kinney JN, Kerfeld CA: Cyanobacterial-based approaches to improving photosynthesis in plants. Journal of Experimental Botany 2013, 64:787798.

* 19. Kinney JN, Salmeen A, Cai F, Kerfeld CA: Elucidating Essential Role of Conserved Carboxysomal Protein CcmN Reveals Common Feature of Bacterial Microcompartment Assembly. J Biol Chem 2012, 287:17729-17736.

*First demonstration that a small peptide segment, the encapsulation peptide, is essential for carboxysome formation, specifically for interaction with the shell, and that peptides with similar properties are widespread among BMCs.

* 20. Cai F, Dou Z, Bernstein SL, Leverenz R, Williams EB, Heinhorst S, Shively JM, Cannon GC, Kerfeld CA: Advances in Understanding Carboxysome Assembly in Prochlorococcus and Synechococcus Implicate CsoS2 as a Critical Component. Life 2015, 5:1141-1171.

* This paper includes a wealth of structural, functional, and evolutionary findings about an intrinsically-disordered protein which has been difficult to characterize, discusses carboxysome assembly, and includes a phylogenetic tree supporting the alpha cyanobacteria as recipients of horizontally-acquired alpha carboxysome genes from chemoautotrophic proteobacteria.

21. Baker SH, Lorbach SC, Rodriguez-Buey M, Williams DS, Aldrich HC, Shively JM: The correlation of the gene csoS2 of the carboxysome operon with two polypeptides of the carboxysome in Thiobacillus neapolitanus. Arch.Microbiol. 1999, 172 233239.

22. Heinhorst S, Cannon GC, Shively JM: Carboxysomes and Carboxysome-like Inclusions. In Microbiol. Monogr., edn 20 May 2006. Edited by; 2006.

23. Chaijarasphong T, Nichols RJ, Kortright KE, Nixon CF, Teng PK, Oltrogge LM, Savage DF: Programmed Ribosomal Frameshifting Mediates Expression of the $\alpha-$ Carboxysome. Journal of Molecular Biology 2015, 428:153-164.

* 24. Roberts EW, Cai F, Kerfeld CA, Cannon GC, Heinhorst S: Isolation and characterization of the Prochlorococcus carboxysome reveal the presence of the novel shell protein CsoS1D. Journal of Bacteriology 2012, 194:787-795.

* This paper not only revealed a new subtype of shell protein for alpha carboxysomes, but established the concept of a carboxysome "superlocus" and describes some of the accessory genes that are conserved in the context of ecotype.

25. Cai F, Sutter M, Cameron JC, Stanley DN, Kinney JN, Kerfeld CA: The Structure of CcmP, a Tandem Bacterial Microcompartment Domain Protein from the betaCarboxysome, Forms a Subcompartment Within a Microcompartment. Journal of Biological Chemistry 2013, 288:16055-16063.

26. Menon BB, Dou Z, Heinhorst S, Shively JM, Cannon GC: Halothiobacillus neapolitanus carboxysomes sequester heterologous and chimeric RubisCO species. PLOS ONE 2008, 3:e3570. 
** 27. Iancu CV, Morris DM, Dou Z, Heinhorst S, Cannon GC, Jensen GJ: Organization, structure, and assembly of a-carboxysomes determined by electron

cryotomography of intact cells. Journal of Molecular Biology 2010, 396:105-117.

**Pioneering study of carboxysome assembly showing development of carboxysomes through interaction of shell proteins and RuBisCO.

28. Baker SH, Williams DS, Aldrich HC, Gambrell AC, Shively JM: Identification and localization of the carboxysome peptide CsoS3 and its corresponding gene in Thiobacillus neapolitanus. Arch Microbiol 2000, 173:278-283.

29. Heinhorst S, Williams EB, Cai F, Murin CD, Shively JM, Cannon GC: Characterization of the carboxysomal carbonic anhydrase CsoSCA from Halothiobacillus neapolitanus. Journal of Bacteriology 2006, 188:8087-8094.

30. So AK-C, Espie GS, Williams EB, Shively JM, Heinhorst S, Cannon GC: A novel evolutionary lineage of carbonic anhydrase (epsilon class) is a component of the carboxysome shell. Journal of Bacteriology 2004, 186:623-630.

31. Kovacs D, Szabo B, Pancsa R, Tompa $P$ : Intrinsically disordered proteins undergo and assist folding transitions in the proteome. Archives of Biochemistry and Biophysics 2013, 531:80-89.

32. Tompa P: Intrinsically disordered proteins: a 10-year recap. Trends in Biochemical Sciences 2012, 37:509-516.

33. Long BM, Badger MR, Whitney SM, Price GD: Analysis of carboxysomes from Synechococcus PCC7942 reveals multiple Rubisco complexes with carboxysomal proteins CcmM and CcaA. Journal of Biological Chemistry 2007, 282:29323-29335.

34. Cameron JC, Wilson SC, Bernstein SL, Kerfeld CA: Biogenesis of a bacterial organelle: the carboxysome assembly pathway. Cell 2013, 155:1131-1140.

35. Ludwig M, Sültemeyer D, Price GD: Isolation of ccmKLMN genes from the marine cyanobacterium, Synechococcus sp. PCC7002 (Cyanophyceae), and evidence that CcmM is essential for carboxysome assembly. Journal of Phycology 2000, 36:11091119.

36. Chen AH, Robinson-Mosher A, Savage DF, Silver Pa, Polka JK: The Bacterial CarbonFixing Organelle Is Formed by Shell Envelopment of Preassembled Cargo. PLoS ONE 2013, 8:1-13.

37. Aussignargues C, Paasch BC, Gonzalez-Esquer R, Erbilgin O, Kerfeld CA: Bacterial microcompartment assembly: The key role of encapsulation peptides. Commun Integr Biol 2015, 8:e1039755.

38. Fan C, Cheng S, Sinha S, Bobik TA: Interactions between the termini of lumen enzymes and shell proteins mediate enzyme encapsulation into bacterial microcompartments. Proc Natl Acad Sci U S A 2012, 109:14995-15000.

39. Marsh Joseph A, Hernández H, Hall Z, Ahnert Sebastian E, Perica T, Robinson Carol V, Teichmann Sarah A: Protein Complexes Are under Evolutionary Selection to Assemble via Ordered Pathways. Cell 2013, 153:461-470.

40. Levy ED, Erba EB, Robinson CV, Teichmann SA: Assembly reflects evolution of protein complexes. Nature 2008, 453:1262-1265.

41. Rae BD, Long BM, Badger MR, Price GD: Functions, compositions, and evolution of the two types of carboxysomes: polyhedral microcompartments that facilitate $\mathrm{CO} 2$ fixation in cyanobacteria and some proteobacteria. Microbiol Mol Biol Rev 2013, 77:357-379.

42. Gupta RS: Origin and spread of photosynthesis based upon conserved sequence features in key bacteriochlorophyll biosynthesis proteins. Molecular Biology and Evolution 2012, 29:3397-3412.

43. Baker SH, Jin S, Aldrich HC, Howard GT, Shively JM: Insertion Mutation of the Form I cbbL Gene Encoding Ribulose Bisphosphate Carboxylase/Oxygenase (RuBisCO) 
in Thiobacillus neapolitanus Results in Expression of Form II RuBisCO, Loss of Carboxysomes, and an Increased CO2 Requirement for Growth. Journal of Bacteriology 1998, 180:4133-4139.

44. Pierce J, Carlson TJ, Williams JG: A cyanobacterial mutant requiring the expression of ribulose bisphosphate carboxylase from a photosynthetic anaerobe. Proceedings of the National Academy of Sciences 1989, 86:5753-5757.

45. Amichay D, Levitz R, Gurevitz M: Construction of a Synechocystic PCC6803 mutant suitable for the study of variant hexadecameric ribulose bisphosphate carboxylase/oxygenase enzymes. Plant Molecular Biology 1993, 23:465-476.

46. Shih PM, Occhialini A, Cameron JC, Andralojc PJ, Parry MAJ, Kerfeld CA: Biochemical characterization of predicted Precambrian RuBisCO. Nature Communications 2016, 7:10382.

47. Soo RM SC, Sekiguchi Y, Imelfort M, Paech SJ, Dennis PG, Steen JA, Parks DH, Tyson GW, Hugenholtz P.: An expanded genomic representation of the phylum Cyanobacteria. Genome Biology and Evolution 2014, 6:1031-1045.

48. Zhaxybayeva O, Gogarten JP, Charlebois RL, Doolittle WF, Papke RT: Phylogenetic analyses of cyanobacterial genomes: quantification of horizontal gene transfer events. Genome Research 2006, 16:1099-1108.

49. Criscuolo A, \& Gribaldo, S. : Large-scale phylogenomic analyses indicate a deep origin of primary plastids within cyanobacteria. Molecular Biology and Evolution 2011, 28:3019-3032.

50. Shih PM, Wu D, Latifi A, Axen SD, Fewer DP, Talla E, Calteau A, Cai F, Tandeau de Marsac N, Rippka R, et al.: Improving the coverage of the cyanobacterial phylum using diversity-driven genome sequencing. Proceedings of the National Academy of Sciences 2013, 110:1053-1058.

51. Benzerara K, Skouri-Panet F, Li J, Ferard C, Gugger M, Laurent T, Couradeau E, Ragon M, Cosmidis J, Menguy N, et al.: Intracellular Ca-carbonate biomineralization is widespread in cyanobacteria. Proceedings of the National Academy of Sciences 2014, 111:10933-10938.

52. Tomitani A, Knoll AH, Cavanaugh CM, Ohno T: The evolutionary diversification of cyanobacteria: molecular-phylogenetic and paleontological perspectives. Proceedings of the National Academy of Sciences 2006, 103:5442-5447.

53. Fischbach M, Voigt CA: Prokaryotic gene clusters: a rich toolbox for synthetic biology. Biotechnology Journal 2010, 5:1277-1296.

54. Bonacci W, Teng PK, Afonso B, Niederholtmeyer H, Grob P, Silver PA, Savage DF: Modularity of a carbon-fixing protein organelle. Proc Natl Acad Sci U S A 2012, 109:478-483.

55. Romero PA, Arnold FH: Exploring protein fitness landscapes by directed evolution. Nat Rev Mol Cell Biol 2009, 10:866-876.

56. Tcherkez GGB, Farquhar GD, Andrews TJ: Despite slow catalysis and confused substrate specificity, all ribulose bisphosphate carboxylases may be nearly perfectly optimized. Proceedings of the National Academy of Sciences 2006, 103:7246-7251.

** 57. Lin MT, Occhialini A, Andralojc PJ, Devonshire J, Hines KM, Parry MA, Hanson MR: beta-Carboxysomal proteins assemble into highly organized structures in Nicotiana chloroplasts. Plant J 2014, 79:1-12.

${ }^{* *}$ Assembly of shell components and demonstration of targeting using the encapsulation peptide in chloroplasts.

58. Lin MT, Occhialini A, Andralojc PJ, Parry MAJ, Hanson MR: A faster Rubisco with potential to increase photosynthesis in crops. Nature 2014, 513:547-550. 
59. Gonzalez-Esquer CR, Shubitowski TB, Kerfeld CA: Streamlined Construction of the Cyanobacterial CO2-Fixing Organelle via Protein Domain Fusions for Use in Plant Synthetic Biology. Plant Cell 2015, 27:2637-2644.

60. Du J, Förster B, Rourke L, Howitt SM, Price GD: Characterisation of Cyanobacterial Bicarbonate Transporters in E. coli Shows that SbtA Homologs Are Functional in This Heterologous Expression System. PLOS ONE 2014, 9:e115905.

61. Tsai Y, Sawaya MR, Cannon GC, Cai F, Williams EB, Heinhorst S, Kerfeld CA, Yeates TO: Structural Analysis of CsoS1A and the Protein Shell of the Halothiobacillus neapolitanus Carboxysome. PLoS Biol 2007, 5:e144.

62. Cai F, Sutter M, Bernstein SL, Kinney JN, Kerfeld CA: Engineering Bacterial Microcompartment Shells: Chimeric Shell Proteins and Chimeric Carboxysome Shells. ACS Synthetic Biology 2015, 4:444-453.

63. He Z, Xu M, Wu Y, Lv J, Fu P, Mi H: NdhM is required for the stability and the function of NAD(P)H dehydrogenase complexes involved in $\mathrm{CO} 2$ uptake in Synechocystis sp. strain PCC 6803. Journal of Biological Chemistry 2015:doi:10.1074/jbc.M1115.698084.

64. Maeda S-i, Badger MR, Price GD: Novel gene products associated with NdhD3/D4containing NDH-1 complexes are involved in photosynthetic $\mathrm{CO} 2$ hydration in the cyanobacterium, Synechococcus sp. PCC7942. Molecular microbiology 2002, 43:425-435.

65. Wheatley NM, Sundberg CD, Gidaniyan SD, Cascio D, Yeates TO: Structure and Identification of a Pterin Dehydratase-like Protein as a Ribulose-bisphosphate Carboxylase/Oxygenase (RuBisCO) Assembly Factor in the $\alpha$-Carboxysome. Journal of Biological Chemistry 2014, 289:7973-7981.

66. Feiz L, Williams-Carrier R, Belcher S, Montano M, Barkan A, Stern DB: A protein with an inactive pterin-4a-carbinolamine dehydratase domain is required for Rubisco biogenesis in plants. The Plant Journal 2014, 80:862-869.

67. Mueller-Cajar O, Stotz M, Wendler P, Hartl FU, Bracher A, Hayer-Hartl M: Structure and function of the AAA+ protein CbbX, a red-type Rubisco activase. Nature 2011, 479:194-199.

68. Battchikova N, Eisenhut M, Aro EM: Cyanobacterial NDH-1 complexes: novel insights and remaining puzzles. Biochim Biophys Acta 2011, 1807:935-944.

69. Samborska B, Kimber Matthew S: A Dodecameric CcmK2 Structure Suggests $\beta$ Carboxysomal Shell Facets Have a Double-Layered Organization. Structure 2012, 20:1353-1362.

70. Tanaka S, Sawaya MR, Phillips M, Yeates TO: Insights from multiple structures of the shell proteins from the $\beta$-carboxysome. Protein Science 2009, 18:108-120.

71. Sutter M, Wilson SC, Deutsch S, Kerfeld CA: Two new high-resolution crystal structures of carboxysome pentamer proteins reveal high structural conservation of $\mathrm{CcmL}$ orthologs among distantly related cyanobacterial species. Photosynthesis Research 2013, 118:9-16.

72. Keeling TJ, Samborska B, Demers RW, Kimber MS: Interactions and structural variability of $\boldsymbol{\beta}$-carboxysomal shell protein $\mathbf{C c m L}$. Photosynthesis Research 2014 , 121:125-133.

* 73. Long BM, Tucker L, Badger MR, Price GD: Functional cyanobacterial betacarboxysomes have an absolute requirement for both long and short forms of the CcmM protein. Plant physiology 2010, 153:285-293.

* This paper demonstrates that domains mimicking the small subunit of RuBisCO are essential for carboxysome formation. 
74. Pena KL, Castel SE, de Araujo C, Espie GS, Kimber MS: Structural basis of the oxidative activation of the carboxysomal carbonic anhydrase, $\mathrm{CcmM}$. Proceedings of the National Academy of Sciences 2010, 107:2455-2460.

75. Marco E, Martinez I, Ronen-Tarazi M, Orus MI, Kaplan A: Inactivation of ccmO in Synechococcus sp. Strain PCC 7942 Results in a Mutant Requiring High Levels of CO2. Applied and Environmental Microbiology 1994, 60:1018-1020.

** 76. Rae BD, Long BM, Badger MR, Price GD: Structural determinants of the outer shell of $\beta$-carboxysomes in Synechococcus elongatus PCC 7942: Roles for CcmK2, K3K4, CcmO, and CcmL. PLOS ONE 2012, 7:14-19.

** This paper presents growth phenotypes and electron micrographs of mutants deficient in several different components, and proposed the minimal structural determinants for the outer shell of beta carboxysomes.

77. Newman J, Branden C-I, Jones TA: Structure determination and refinement of ribulose 1,5-bisphosphate carboxylase/oxygenase from Synechococcus PCC6301. Acta Crystallographica Section D 1993, 49:548-560.

78. Saschenbrecker S, Bracher A, Rao KV, Rao BV, Hartl FU, Hayer-Hartl M: Structure and Function of RbcX, an Assembly Chaperone for Hexadecameric Rubisco. Cell 2007, 129:1189-1200.

79. Tanaka S, Sawaya MR, Kerfeld CA, Yeates TO: Structure of the RuBisCO chaperone RbcX from Synechocystis sp. PCC6803. Acta Crystallographica Section D 2007, 63:1109-1112.

80. Cot SS-W, So AK-C, Espie GS: A Multiprotein Bicarbonate Dehydration Complex Essential to Carboxysome Function in Cyanobacteria. Journal of Bacteriology 2008, 190:936-945.

81. So A, John-McKay M, Espie G: Characterization of a mutant lacking carboxysomal carbonic anhydrase from the cyanobacterium Synechocystis PCC6803. Planta 2002, 214:456-467. 
Table 1: Genes conserved in carboxysome superloci.

Symbol

in 2

Name

Description

PFAM

Function

Alpha Carboxysome:

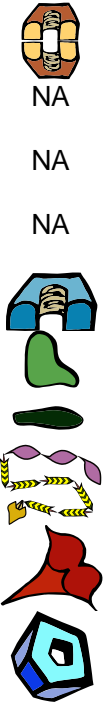

CsoS1D BMC-T Protein: stacked and gated shell protein

Ham1 HAM1-family Protein (alpha-NTPPyrophosphatase family)

SbtA Sodium-dependent Bicarbonate Component: PII-like

CsoS1 BMC-H Protein: CsoS1

CbbL Form 1A RuBisCO Large Subunit

CbbS

Csos2

Form 1A RuBisCO Small Subunit

Conserved Carboxysome Protein: Csos2

CsoSCA Beta-class Carbonic Anhydrase

BMC-P Protein

BMC-H Protein, with extension

CsoS1E

NA

NA

$\mathrm{Bfr}$

NdhF

Bacterioferritin homolog

$\mathrm{NDH}-1$ Complex, Subunit 5 (chain L)

NA

$\mathrm{NdhD}$

NDH-1 Complex, Subunit 4 (chain M)

NA ChpXY

$\mathrm{CO}_{2}$ Hydration Protein (CupB)

NA PCD-like

Pterin-4-alpha-Carbinolamine Dehydratase homolog / acRAF

NA

CbbX

PF00936

PF01725

PF05982

PF00543

PF00936

PF02788/

PF00016

PF00101

PF12288

PF08936

PF03319

PF03319

PF00936

PF00210

PF00361/

PF00662

PF00361

PF10216

PF01329 RuBisCO Activase Transporter

SbtB Bicarbonate Transport Regulatory

BMC-P Protein

Shell vertex

Shell $(B M C-H)$ ?

\section{Beta Carboxysome:}

NA ChpXY $\quad \mathrm{CO}_{2}$ Hydration Protein (CupB)

NA NdhM

NA NdhF3

$\mathrm{CcmK} 1$

CcmK2

$\mathrm{CcmL}$

CcmM

$\mathrm{CcmN}$

$\mathrm{CcmO}$

CcmK3

CcmK4

$\mathrm{CcmP}$

$\mathrm{RbcL}$

$\operatorname{RbcX}$

$$
\mathrm{NDH}-1 \text { Complex Subunit }
$$

$\mathrm{NAD}(\mathrm{P}) \mathrm{H}$ Dehydrogenase Subunit (NdhF3 family) BMC-H Protein

BMC-H Protein

BMC-P Protein

Gamma-class Carbonic Anhydrase / RuBisCO Nucleation Protein

Hexapeptide Repeat Protein with Encapsulation Peptide

BMC-T Protein

BMC-H Protein

BMC-H Protein

BMC-T Protein: stacked and gated shell protein

NA

RbcS

Form 1B RuBisCO Large Subunit

Chaperonin Family Protein: RbcX

Form 1B RuBisCO Small Subunit

CcaA

Beta-class Carbonic Anhydrase
PF10216

PF01059/

PF00361

PF00361/

PF00662

PF00936

PF00936

PF03319

PF00132/

PF14602/

PF00101

PF00132

PF00936

PF00936

PF00936

PF00936

PF02788/ PF00016

PF02341

PF00101

PF00484
Unknown

$\mathrm{CO}_{2}$ uptake?

$\mathrm{CO}_{2}$ uptake?

$\mathrm{CO}_{2}$ uptake?

RubisCO assembly?

RuBisCO assembly

$\mathrm{CO}_{2}$ uptake?

$\mathrm{CO}_{2}$ uptake?

$\mathrm{CO}_{2}$ uptake?

Shell

Shell

Shell vertex

$\mathrm{CO}_{2}$ generation/ carboxysome assembly

Carboxysome assembly

Shell

Shell

Shell

Shell

$\mathrm{CO}_{2}$ fixation

RuBisCO assembly

$\mathrm{CO}_{2}$ fixation

$\mathrm{CO}_{2}$ generation
Distribution

Locus Type

("Picocyanobacterial Clades" per Shih et al. 2013)

Superlocus All alpha cyanobacteria

$[3,12,24]$

Superlocus

All alpha cyanobacteria

CB4 \& CB5 clades, C. gracile sub-cluster

CB4 \& CB5 clades, C. gracile sub-cluster

All alpha cyanobacteria

Core

All alpha cyanobacteria

All alpha cyanobacteria

All alpha cyanobacteria

$[20,21,23]$

All alpha cyanobacteria

Core

Core

All alpha cyanobacteria

All alpha cyanobacteria

All alpha cyanobacteria except high light-adapted Prochlorococcus

Superlocus

Superlocus

Superlocus

Superlocus

Superlocus

Superlocus

Sub-clusters 5.2, 5.3, \& $6 \mathrm{~b}$

Sub-clusters 5.1 (A/B), 5.2, 5.3, $\& 6 b$

Sub-clusters 5.1 (A/B), 5.2, 5.3, $\& 6 \mathrm{~b}$

Sub-clusters $5.1(\mathrm{~A} / \mathrm{B})$, 5.2, 5.3, $\& 6 b$

All alpha cyanobacteria and chemoautotrophs

Sub-clusters 5.1 (A/B), 5.2, 5.3, $\& 6 b$

Superlocus

Majority of beta cyanobacteria

Superlocus

Majority of beta cyanobacteria

$[3,63,68]$

Superlocus

Majority of beta cyanobacteria

$[3,68]$

Core

Majority of beta cyanobacteria

All beta cyanobacteria

$[14,15,19,62$

$, 69,70]$

All beta cyanobacteria

$[14,71,72]$

Core

All beta cyanobacteria

Core

All beta cyanobacteria

[19]

Core/Satellite

All beta cyanobacteria

Satellite

Beta cyanobacteria

[76]

Satellite

Satellite

Beta cyanobacteria

Beta cyanobacteria

$[15,62,76]$

[25]

Core/Satellite

All beta cyanobacteria

[77]

Core/Satellite

All beta cyanobacteria

$[78,79]$

Core/Satellite

All beta cyanobacteria

Satellite 
